\title{
GUST RESPONSE OF A TWO-DIMENSIONAL NONLINEAR WING
}

\author{
Xiaoyang Zhang, Mojtaba Kheiri, Wen-Fang Xie \\ Department of Mechanical, Industrial and Aerospace Engineering \\ Concordia University \\ Montreal, QC, Canada \\ xiaoyang.zhang@mail.concordia.ca,mojtaba.kheiri@concordia.ca,wfxie@encs.concordia.ca
}

\begin{abstract}
In this paper, the equations of motion for a twodimensional (2-D) wing encountering a gust are presented. The wing has two degrees of freedom, i.e. pitch and plunge, and its motion is restrained by nonlinear translational and rotational springs. Two different stiffness nonlinearities are examined: (i) cubic and (ii) free play. For given system parameters, the responses of the nonlinear system to the sharp-edged and 1cosine gust profiles are obtained at different flow velocities and compared to the time response of the system with no gust input.
\end{abstract}

Keywords: aeroelasticity; gust response; cubic nonlinearity; freeplay nonlinearity; flutter analysis

\section{INTRODUCTION}

Aeroelasticity is an interdisciplinary field of study dealing with interactions between inertia, aerodynamic and elastic forces. Classical theories in aeroelasticity assume linear aerodynamics and structural dynamics resulting in a set of linear equations. These equations can be fairly easily solved in time or frequency domain and used for examining the aircraft stability and response to external excitation. However, linear aerodynamic theories start to break down at high airspeed. Flow separation and shock oscillations may also introduce aerodynamic nonlinearities. Moreover, structural nonlinearities may arise from, for example, large deformations, material behavior, worn hinges of control surfaces, and loose control linkages $[1,2]$.

Woolston et al. may be the first researchers that investigated the effects of structural nonlinearities on the flutter of a wing [1]. Two different wing models were studied: a wing capable of bending and twisting (i.e. two-degree-of-freedom (2-DOF) system) and, also, a wing with a control surface (i.e. 3-DOF system). They considered structural nonlinearities as concentrated in the torsional stiffness. Three different types of nonlinear springs, namely flat spot (representing free play), hysteresis, and cubic were examined. They found that there is a strong connection between the stability of a nonlinear system and the initial conditions - in many cases the flutter speed was decreased as the initial disturbance was increased.

A comprehensive review of different types of structural and aerodynamic nonlinearities encountered in aeronautical engineering was conducted by Lee et al. [2]. They discussed several techniques, such as finite difference and describing function, for solving equations with structural nonlinearities. They found that even a 2-DOF system with a single nonlinearity in the pitch degree of freedom may show a complex dynamical behavior. For example, they showed that a system with a freeplay nonlinearity may undergo period-1, period-2, and period-4 limit-cycle oscillations (LCO), as well as, chaotic motion, depending on the airspeed. For a more recent and quite shorter review of nonlinear aeroelasticity, the reader is referred to [3].

Several researchers have studied the response of a lifting surface to a time-dependent external excitation. For example, Poirel and Price investigated the effect of longitudinal atmospheric turbulence on the dynamics of an airfoil with a hardening cubic structural nonlinearity in pitch [4]. They found that flutter occurred at a lower velocity for the excited case than the so-called non-excited one, whereas the onset of LCO occurred at a higher velocity. Marzocca et al. investigated the aeroelastic response to a time-dependent external excitation of a 2-D rigid/elastic-lifting surface in incompressible flow field, featuring plunging-pitching coupled motion [5]. Haddadpour et al. examined the effects of the sharp-edged gust on the dynamics of a flexible high-aspect ratio wing [6]. In their model, they used linear structural dynamics and linear quasi-steady aerodynamics.

In reality, almost all lifting surfaces involve some sort of structural nonlinearity, and they may also encounter with atmospheric turbulence during a normal flight. Nevertheless, very few studies on the nonlinear aeroelastic response to a timedependent excitation can be found in the body of the literature. This is, in fact, the principal objective of this paper, namely studying the effects of a time-dependent excitation, such as sharp-edge and 1-cosine gusts on the dynamics and stability of a 2-DOF typical airfoil section with structural nonlinearities.

\section{NONLINEAR AEROELASTIC MODEL WITH GUST EXCITATION}

\section{A. Governing Equations Including the Gust Input}

Fig. 1 shows the cross-section of a 2-D rigid wing with degrees of freedom in the plunge and pitch directions. The plunge displacement is measured from the elastic axis and is represented by $h$ (positive downward); $\alpha$ is the pitch angle about the elastic axis (positive nose up). The elastic axis is located at a distance $a b$ from the mid-chord, while the mass center is located at a distance $x_{\alpha} b$ from the elastic axis, $b$ being the semi-chord. The wing is supported by a translational and a rotational spring, attached to the elastic axis, which generally have a nonlinear stiffness. 


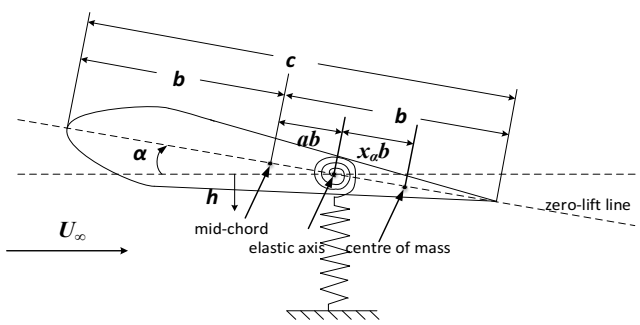

Figure 1. Schematic showing geometry of the wing section.

Here, we follow formulations presented in $[2,5]$. The dimensionless aeroelastic equations featuring coupled plungingpitching motion of a typical airfoil section exposed to a gust input are expressed as

$$
\begin{gathered}
\xi^{\prime \prime}+x_{\alpha} \alpha^{\prime \prime}+2 \zeta_{\xi} \frac{\bar{\omega}}{U^{*}} \xi^{\prime}+\left(\frac{\bar{\omega}}{U^{*}}\right)^{2} G(\xi)-l_{a}(\tau)=l_{g}(\tau), \\
\left(\frac{x_{\alpha}}{r_{\alpha}}\right) \xi^{\prime \prime}+\alpha^{\prime \prime}+2 \zeta_{\alpha} \frac{1}{U^{*}} \alpha^{\prime}+\frac{1}{U^{* 2}} M(\alpha)-m_{a}(\tau)=m_{g}(\tau),
\end{gathered}
$$

where $\xi=h / b$ is the dimensionless plunge displacement, and $\zeta_{\xi}$ and $\zeta_{\alpha}$ are, respectively, viscous damping ratios in pitch and plunge; $U^{*}=U_{\infty} / b \omega_{\alpha}$ is the dimensionless velocity, and $\bar{\omega}=$ $\omega_{\xi} / \omega_{\alpha}$ is the frequency ratio, $U_{\infty}$ being the freestream velocity, $\omega_{\xi}$ and $\omega_{\alpha}$ also being natural frequencies in pitch and plunge, respectively; also, $l_{a}(\tau)$ and $m_{a}(\tau)$ are the aerodynamic lift and pitching moment about the elastic axis, respectively, while $l_{g}(\tau)$ and $m_{g}(\tau)$ are the lift and moment about the elastic axis due to the gust; $G(\xi)$ and $M(\alpha)$ represent the nonlinear plunge and pitch structural stiffness terms, respectively; moreover, ()$^{\prime} \equiv$ $\partial() / \partial \tau$ denotes the time derivative with respect to dimensionless time $\tau$.

The aerodynamic lift $l_{a}(\tau)$ and pitching moment $m_{a}(\tau)$ may be expressed as

$$
\begin{gathered}
l_{a}(\tau)=-\frac{2}{\mu} \int_{0}^{\infty} \phi(\tau-\sigma)\left(\alpha^{\prime}+\xi^{\prime \prime}+\left(\frac{1}{2}-a\right) \alpha^{\prime \prime}\right) d \sigma- \\
\frac{1}{\mu}\left(\alpha^{\prime}+\xi^{\prime \prime}-a \alpha^{\prime \prime}\right), \\
m_{a}(\tau)=\frac{2}{\mu}\left(\frac{1}{2}+a\right) \int_{0}^{\infty} \phi(\tau-\sigma)\left(\alpha^{\prime}+\xi^{\prime \prime}+\left(\frac{1}{2}-a\right) \alpha^{\prime \prime}\right) d \sigma \\
+\frac{1}{r_{\alpha}^{2} \mu} a\left(\xi^{\prime \prime}-a \alpha^{\prime \prime}\right)-\frac{1}{r_{\alpha}^{2} \mu}\left(\frac{1}{2}-a\right) \alpha^{\prime}-\frac{1}{8} \frac{1}{r_{\alpha}^{2} \mu} \alpha^{\prime \prime},
\end{gathered}
$$

where $\mu=m / \pi \rho b^{2}$ is the mass ratio, and $r_{\alpha}=I_{\alpha} / m b^{2}$ is the dimensionless radius of gyration; $m, \rho$, and $I_{\alpha}$ being, respectively, mass of the wing per unit span, air density, and mass moment of inertia per unit span. Also, $\phi(\tau)$ is called the Wagner function and is given by

$$
\phi(\tau)=1-A_{1} e^{-b_{1} \tau}-A_{2} e^{-b_{2} \tau},
$$

where the constants are $A_{1}=0.165, A_{2}=0.335, b_{1}=$ $0.0455, b_{2}=0.3$.

By considering $w_{G}(\tau)$ as a gust variable velocity and using Duhamel's integral concept, the lift and pitching moment about the elastic axis due to the penetration into the gust, $l_{g}(\tau)$ and $m_{g}(\tau)$, respectively, may be written as

$$
\begin{gathered}
l_{g}(\tau)=\frac{2}{\mu} \int_{0}^{\tau} \psi^{\prime}(\tau-\sigma) \frac{w_{G}(\tau)}{U_{\infty}} d \sigma \\
m_{g}(\tau)=\left(\frac{1}{2}+a\right) \frac{2}{r_{\alpha}^{2} \mu} \int_{0}^{\tau} \psi^{\prime}(\tau-\sigma) \frac{w_{G}(\tau)}{U_{\infty}} d \sigma
\end{gathered}
$$

where $\psi(\tau)$ is called the Küssner function.

A widely-used approximation for Küssner's function is the following two-term exponential expression [2]:

$$
\psi(\tau)=1-A_{3} e^{-b_{3} \tau}-A_{4} e^{-b_{4} \tau},
$$

where the constants are $A_{3}=0.5, A_{4}=0.5, b_{3}=0.130$ and $b_{4}=1$.

In this paper, two different gust profiles, implying specific time variations of their velocity distribution, will be used. They are: (a) sharp-edged gust, and (b) 1-cosine gust. Their analytical expressions are:

$$
\begin{gathered}
\text { sharp - edged gust: } w_{G}(\tau)=H(\tau) w_{0}, \\
1-\text { cosine gust: } w_{G}(\tau)=H(\tau) \frac{1}{2} w_{0}\left(1-\cos \frac{\pi \tau}{\tau_{G}}\right) \\
-H\left(\tau-2 \tau_{G}\right) \frac{1}{2} w_{0}\left(1-\cos \frac{\pi \tau}{\tau_{G}}\right),
\end{gathered}
$$

where $w_{0}$ is the gust maximum amplitude, and $H(\tau)$ represents the Heaviside step function.

\section{B. State-space Equations}

In order to deal with the integral terms in (3-4) and (6-7), we introduce six new variables or states as

$$
\begin{array}{r}
w_{1}=\int_{0}^{\tau} e^{-b_{1}(\tau-\sigma)} \alpha(\sigma) d \sigma, \quad w_{2}=\int_{0}^{\tau} e^{-b_{2}(\tau-\sigma)} \alpha(\sigma) d \sigma, \\
w_{3}=\int_{0}^{\tau} e^{-b_{1}(\tau-\sigma)} \xi(\sigma) d \sigma, \quad w_{4}=\int_{0}^{\tau} e^{-b_{2}(\tau-\sigma)} \xi(\sigma) d \sigma, \\
w_{5}=\int_{0}^{\tau} e^{-b_{3}(\tau-\sigma)} \frac{w_{G}(\sigma)}{U_{\infty}} d \sigma, \quad w_{6}=\int_{0}^{\tau} e^{-b_{4}(\tau-\sigma)} \frac{w_{G}(\sigma)}{U_{\infty}} d \sigma .
\end{array}
$$

Using variables in (11), the aeroelastic equations (1-2) may be re-written as

$$
\begin{gathered}
c_{0} \xi^{\prime \prime}+c_{1} \alpha^{\prime \prime}+c_{2} \xi^{\prime}+c_{3} \alpha^{\prime}+c_{4} \xi+c_{5} \alpha+c_{6} w_{1}+c_{7} w_{2} \\
+c_{8} w_{3}+c_{9} w_{4}+c_{10} w_{5}+c_{11} w_{6}+\left(\frac{\bar{\omega}}{U^{*}}\right)^{2} G(\xi)=f(\tau), \\
d_{0} \xi^{\prime \prime}+d_{1} \alpha^{\prime \prime}+d_{2} \xi^{\prime}+d_{3} \alpha^{\prime}+d_{4} \xi+d_{5} \alpha+d_{6} w_{1}+d_{7} w_{2} \\
+d_{8} w_{3}+d_{9} w_{4}+d_{10} w_{5}+d_{11} w_{6}+\left(\frac{1}{U^{*}}\right)^{2} M(\alpha)=g(\tau),
\end{gathered}
$$

where $G(\xi)$ and $M(\alpha)$ are nonlinear functions of $\xi$ and $\alpha$, respectively. $f(\tau)$ and $g(\tau)$ are functions of initial conditions and terms in the Wagner function, which are given by:

$$
\begin{gathered}
f(\tau)=\frac{2}{\mu}\left(\left(\frac{1}{2}-a\right) \alpha(0)+\xi(0)\right)\left(A_{1} b_{1} e^{-b_{1} \tau}+A_{2} b_{2} e^{-b_{2} \tau}\right) \\
g(\tau)=-\frac{(1+2 a) f(\tau)}{2 r_{\alpha}^{2}}
\end{gathered}
$$


The coefficients $c_{i}(i=0, . .9)$ and $d_{i}(i=0, . .9)$ in (12) and (13) were given in [2] and will not be repeated here for the sake of brevity; however, $c_{10}, c_{11}, d_{10}$ and $d_{11}$ are given as

$$
\begin{gathered}
c_{10}=-\frac{2}{\mu} A_{3} b_{3}, c_{11}=-\frac{2}{\mu} A_{4} b_{4}, \\
d_{10}=-\left(\frac{1}{2}+a\right) \frac{2}{r_{\alpha}^{2} \mu} A_{3} b_{3}, d_{11}=-\left(\frac{1}{2}+a\right) \frac{2}{r_{\alpha}^{2} \mu} A_{4} b_{4} .
\end{gathered}
$$

After introducing a variable vector $X=\left(x_{1}, x_{2}, \ldots, x_{10}\right)^{T}$ defined as

$$
\begin{gathered}
x_{1}=\alpha, x_{2}=\alpha^{\prime}, x_{3}=\xi, x_{4}=\xi^{\prime}, x_{5}=w_{1}, x_{6}=w_{2}, \\
x_{7}=w_{3}, x_{8}=w_{4}, x_{9}=w_{5}, x_{10}=w_{6} .
\end{gathered}
$$

(12) and (13) can be written as a set of ten first-order ordinary differential equations.

\section{NUMERICAL EXAMPLES AND ANALYSIS}

The dimensionless parameters of the linear system used in this paper are: $a=-0.5, \mu=100, x_{\alpha}=0.25, r_{\alpha}=0.5, \zeta_{\alpha}=\zeta_{\xi}=$ 0.0 , and $\bar{\omega}=0.2$. We set $\alpha^{\prime}(0)=\xi^{\prime}(0)=\xi(0)=0, \alpha(0)=1 \mathrm{deg}$ as the initial conditions. Using the eigenvalue solution method with the given parameters, we obtain the linear dimensionless flutter speed $\left(U_{L}^{*}\right)$ as 6.28 , which agrees well with results in [2].

Since in this paper we consider nonlinearities only for the pitch DOF, plunging oscillations display very much expected features and are not worthy to be discussed here. Thus, throughout the paper, we only discuss the response in the pitching direction.

\section{A. Gust Response with Cubic Nonlinearity}

We consider a cubic nonlinearity in the structural stiffness for the pitch and a linear stiffness for the plunge degree of freedom. Thus, functions $G(\xi)$ and $M(\alpha)$ in (12) and (13), respectively, can be set to $G(\xi)=\xi$ and $M(\alpha)=\beta_{1} \alpha+\beta_{3} \alpha^{3}$. First, we examined a system with a weak cubic nonlinearity, where $\beta_{1}=1$ and $\beta_{3}=10$. Assuming a sharp-edged gust with $w_{0}=10 \mathrm{~m} / \mathrm{s}$, the response of the system was obtained at $U^{*}=$ 6.27 (not shown here because of space limitation). It was found that the oscillations gradually decay with time, and the system regains its static equilibrium. Thus, even a strong excitation at an airspeed very close to the linear flutter speed cannot change the stability of the system with a weak cubic nonlinearity.

Next, we consider a system with a strong cubic nonlinearity $\left(\beta_{1}=0.01, \beta_{3}=10\right)$ and keep other system parameters the same. The linear flutter speed for this system is $U_{L}^{*}=0.91$. The response of the system to a sharp-edged gust and also a 1-cosine gust with the maximum amplitude of $w_{0}=1 \mathrm{~m} / \mathrm{s}$ at $U^{*}=$ $0.84, U^{*}=U_{L}^{*}=0.91$ and $U^{*}=2 U_{L}^{*}=1.82$ are shown in Figs. 3-5, respectively. Note that for the 1-cosine gust, $\tau_{G}=20$ throughout the paper.

As seen from Fig. 2, both the sharp-edged and 1-cosine gusts result into LCO with comparable amplitudes at $U^{*}=0.84<U_{L}^{*}$, while for the system without the gust input, oscillations decay with time. ${ }^{1}$ This means that the nonlinear system may undergo a Hopf bifurcation leading to $\mathrm{LCO}$ at a speed below the linear flutter speed, provided that a sufficiently strong gust excites it. Fig. 3 shows the dynamic response of the system with and without the gust input at the linear flutter speed, $U_{L}^{*}$. From the figure, one may infer that except for the transient part of the response, the gust does not make any significant changes to the dynamics of the system at $U^{*}=U_{L}^{*}$.

Fig. 4 Shows time responses of the system with and without the gust input at $U^{*}=2 U_{L}^{*}=1.82$. As seen from the figure, the amplitude of oscillations when the system encounters a gust is obviously higher than that when there is no gust. In addition, the system undergoes period-2 motion in response to the gust input, while it performs period-1 motion when there is no gust. It is also interesting to see that the sharp-edged gust tends to increase the pitch angle in the negative direction, whereas the 1-cosine gust does the opposite.
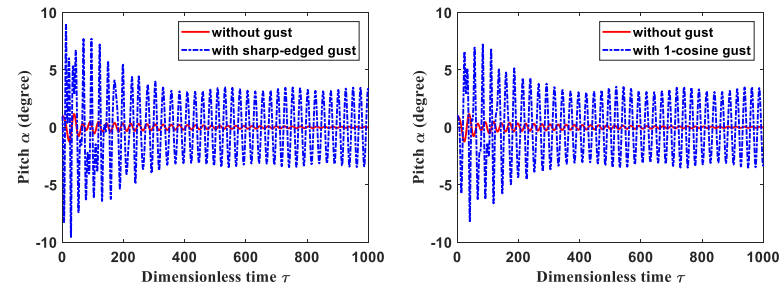

Figure 2. Sharp-edged (left) and 1-cosine (right) gust responses of a system with the strong cubic nonlinearity in pitch at $U^{*}=0.84 ; w_{0}=1 \mathrm{~m} / \mathrm{s}$.
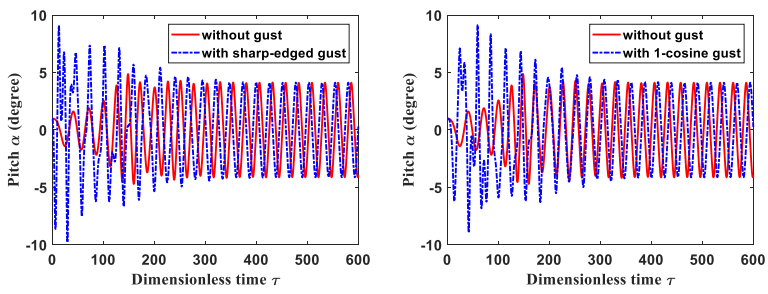

Figure 3. Sharp-edged (left) and 1-cosine (right) gust responses of a system with the strong cubic nonlinearity in pitch at $U^{*}=U_{L}^{*}=0.91 ; w_{0}=1 \mathrm{~m} / \mathrm{s}$.
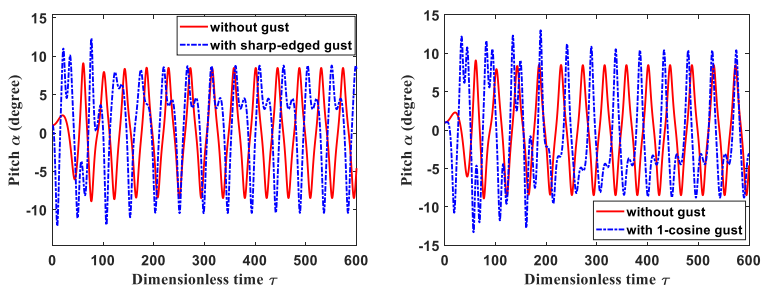

Figure 4. Sharp-edged (left) and 1-cosine (right) gust responses of a system with the strong cubic nonlinearity in pitch at $U^{*}=2 U_{L}^{*}=1.82 ; w_{0}=1 \mathrm{~m} / \mathrm{s}$.

\section{B. Gust Response with Free-play Nonlinearity}

The free-play nonlinearity, in its general form, may be represented by the trilinear stiffness model, as shown in Fig. 5. In the figure, $M_{0}$ represents the preload, $M_{f}$ the stiffness in the free-play zone, $\alpha_{f}$ the pitch angle offset, and $\delta$ the free-play range. We examine the dynamics of a system with free-play

\footnotetext{
${ }^{1}$ The time responses to the gust inputs were obtained for a longer time range (not shown in Fig. 2) where period-1 LCO were observed.
} 
parameters $\delta=0.5^{\circ}, M_{0}=M_{f}=0, \alpha_{f}=-0.25^{\circ}$ for the pitch stiffness. As also previously mentioned, the linear flutter speed for this system is $U_{L}^{*}=6.28$.

Fig. 6 shows the bifurcation diagram for the free vibration (i.e. no gust) of a typical airfoil section with free-play type nonlinearity in pitch, where the peak values of the pitch angle, $\alpha$, are plotted against the normalized dimensionless airspeed, $U^{*} / U_{L}^{*}$. In the bifurcation diagram, a single dot/point represents a static equilibrium position (region I); two dots represent a period-1 motion (regions II and IX), and four dots show a period2 motion (regions III and VIII); also, eight dots represent a period-4 motion (regions V and VII), and finally, multiple scattered dots show a chaotic motion (regions IV and VI). Table I summarizes the dynamical behavior of the system shown in the bifurcation diagram. It is noted that, as seen in Fig. 6, in region IX, the pitch amplitude increases sharply with $U^{*} / U_{L}^{*}$ and goes to very large values for $U^{*} / U_{L}^{*}>1$ (not shown in the figure). These indicate the occurrence of divergent or violent flutter in region IX (for more details, see [1, 2]).

The important message of Fig. 6 is that the free-play nonlinearity may cause the system to lose the static equilibrium at a much lower flow velocity than the linear flutter speed (e.g. at 0.95 instead of 6.28 for the given system parameters). The bifurcation diagram also suggests that the route to chaos is via period-doubling, as also observed by Lee et al. [2].

Fig. 7 shows the typical phase-plane diagrams of four types of motion, which are period-1, period-2, period-4 and chaotic, respectively, at $U^{*} / U_{L}^{*}=0.8,0.56,0.45$, and 0.48 .

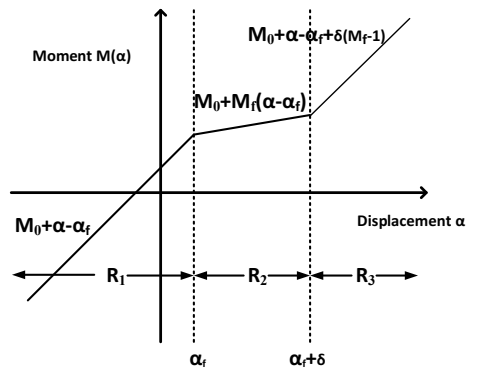

Figure 5. General free-play stiffness model; $M_{0}$ represents the preload; $M_{\mathrm{f}}$ is the stiffness in the free-play zone, $\alpha_{\mathrm{f}}$ is the pitch angle offset, and $\delta$ is the freeplay range.

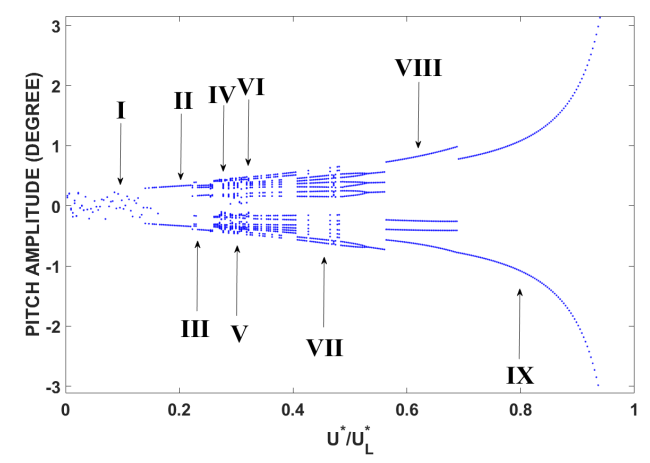

Figure 6. Bifurcation diagram of a system with a free-play nonlinearity in pitch $\left(\delta=0.5^{\circ}, M_{0}=M_{f}=0, \alpha_{f}=-0.25^{\circ}\right)$, where the peak values of the pitch angle is plotted versus the normalized dimensionless airspeed, $U^{*} / U_{L}^{*}$.
TABLE I. DYNAMICAL BEHAVIOUR IN DIFFERENT REGIONS OF THE BIFURCATION DIAGRAM SHOWN IN FIG. $6 ;\left(U^{*} / U_{L}^{*}\right)$ IS THE NORMALIZED DIMENSIONLESS AIRSPEED.

\begin{tabular}{|c|c|c|}
\hline Region & $\left(\boldsymbol{U}^{*} / \boldsymbol{U}_{\boldsymbol{L}}^{*}\right)$ & Type of motion \\
\hline Before I & $(00.151)$ & Static Equilibrium \\
\hline I and VIII & $(0.1510 .221)$ and $(0.6881)$ & Period-1 \\
\hline II and VII & $(0.2210 .255)$ and $(0.5290 .688)$ & Period-2 \\
\hline IV and VI & $(0.3310 .463)$ and $(0.4840 .529)$ & Period-4 \\
\hline III and V & $(0.2550 .331)$ and $(0.4630 .484)$ & Chaos \\
\hline
\end{tabular}
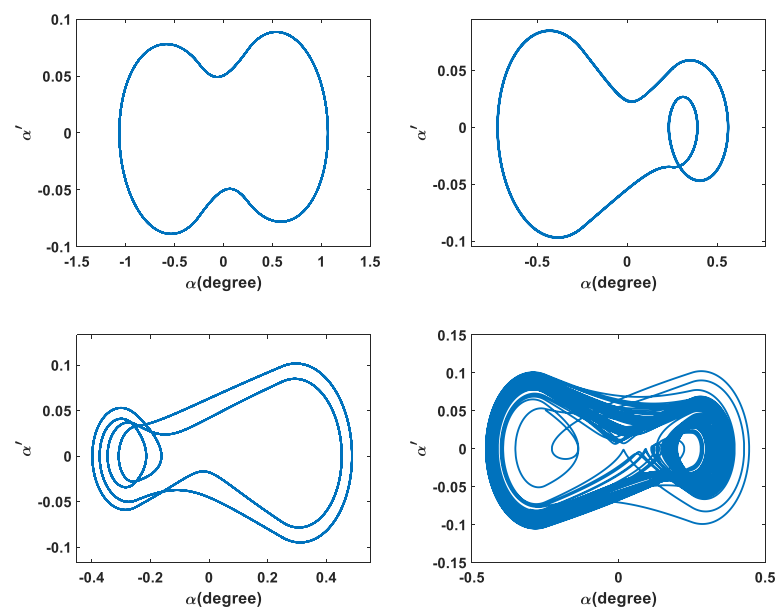

Figure 7. Phase-plane diagrams for different regions shown in Fig. 6; where $U^{*} / U_{L}^{*}=0.8,0.56,0.45$, and 0.48 for the top-left, top-right, bottom-left, and botom-right subfigures, respectively.

Fig. 8 shows time responses of the system in the pitch direction to a sharp-edged (left) and a 1-cosine (right) gust at $U^{*}=0.93$. For comparison purposes, the gust response is plotted over the time response in the absence of the gust. As seen, the system undergoes LCO under the gust input, while in the absence of the gust it regains static stability. In other words, the gust input causes the system to lose its static stability at a lower airspeed, i.e. at $U^{*}=0.87$ instead of 0.95 .

Fig. 9 shows the time responses at $U^{*}=1.30$ which lies in region II in the bifurcation diagram in Fig. 6. As discussed, within this region, the airfoil section undergoes period-1 LCO in the absence of a gust. However, this changes to period-2 LCO when the airfoil encounters a sharp-edged or 1-cosine gust. Thus, the gust may change the type of the oscillatory motion. In addition, it increases the amplitude of oscillation, especially in the transient part of the response.

Fig. 10 shows time responses at $U^{*}=3.06$. Here also, the period-4 motion, which occurs in the gust absence, changes to a chaotic type motion due to the gust input. This is more evident from Fig. 11 which shows the phase-plane plots at the same airspeed without (left) and with (right) a sharp-edged gust input. Fig. 12 shows time responses at $U^{*}=6.00$. Very large amplitudes due to gust effects are noticeable in the transient part of the responses. As seen from Fig. 13, when $U^{*}$ approaches $U_{L}^{*}$, the amplitude of LCO due to the gust input increases rapidly to very large values (of the order of $35 \mathrm{deg}$ ). When $U^{*}$ is greater than $U_{L}^{*}$, namely in the supercritical region, the time responses increase exponentially, indicating divergent flutter (see Fig. 14). 
As seen from the figure, the gust response amplitude is always greater than the one in the absence of the gust.
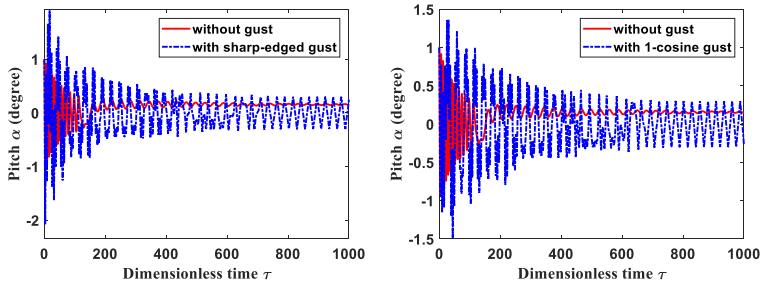

Figure 8. Sharp-edged (left) and 1-cosine (right) gust responses of a system with the free-play nonlinearity in pitch at $U^{*}=0.93 ; w_{0}=1 \mathrm{~m} / \mathrm{s}$.
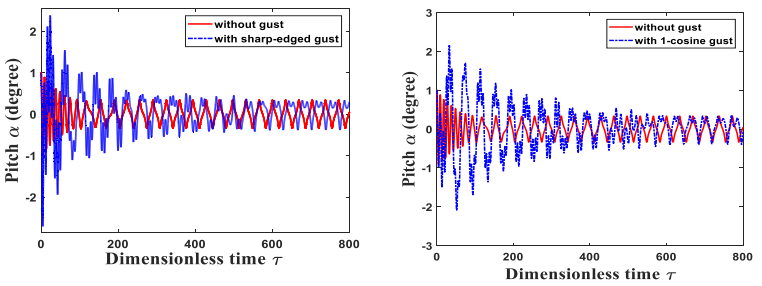

Figure 9. Sharp-edged (left) and 1-cosine (right) gust responses of a system with the free-play nonlinearity in pitch at $U^{*}=1.30 ; w_{0}=1 \mathrm{~m} / \mathrm{s}$.
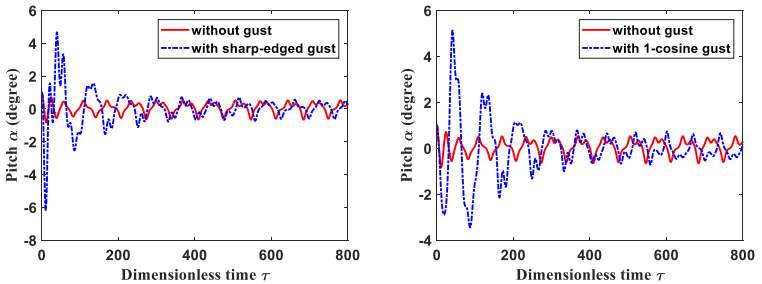

Figure 10. Sharp-edged (left) and 1-cosine (right) gust responses of a system with the free-play nonlinearity in pitch at $U^{*}=3.06 ; w_{0}=1 \mathrm{~m} / \mathrm{s}$.
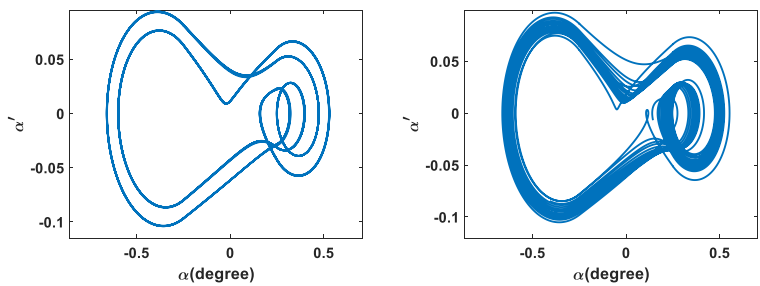

Figure 11. Phase-plane diagrams with gust and without gust (sharp-edged) at $U^{*}=3.06$; without (left) and with (right) a sharp-edged gust, featuring period-4 and chaotic motions, respectively; $w_{0}=1 \mathrm{~m} / \mathrm{s}$.
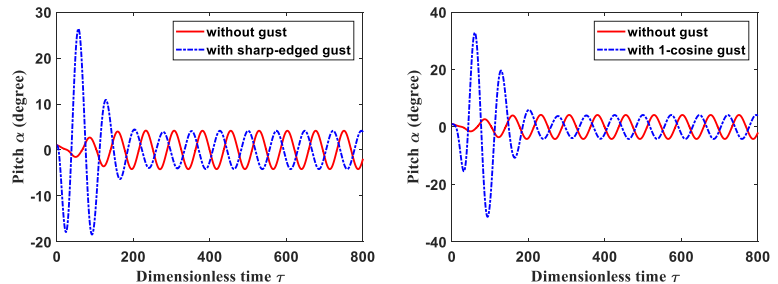

Figure 12. Sharp-edged (left) and 1-cosine (right) gust responses of a system with the free-play nonlinearity in pitch at $U^{*}=6.00 ; w_{0}=1 \mathrm{~m} / \mathrm{s}$.
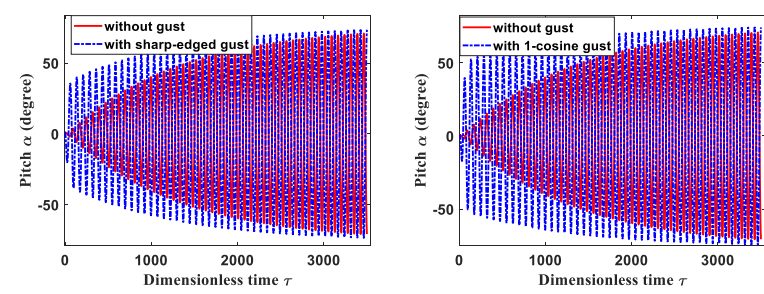

Figure 13. Sharp-edged (left) and 1-cosine (right) gust responses of a system with the free-play nonlinearity in pitch at $U^{*}=6.27 ; w_{0}=1 \mathrm{~m} / \mathrm{s}$.
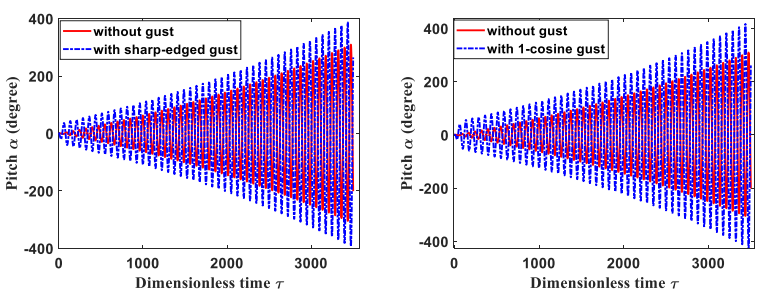

Figure 14. Sharp-edged (left) and 1-cosine (right) gust responses of a system with the free-play nonlinearity in pitch at $U^{*}=6.29 ; w_{0}=1 \mathrm{~m} / \mathrm{s}$.

\section{ACKNOWLEDGMENT}

The authors are grateful to the Faculty of Engineering and Computer Science of Concordia University for the FRS funding. The second author also greatly appreciates the Faculty for a Start-Up grant.

\section{CONCLUDING REMARKS}

The numerical results presented in this paper show that a sharp-edged or a 1-cosine gust input may influence the dynamics and stability of a two-dimensional wing with a strong cubic or free-play stiffness nonlinearity in three different ways: (1) it may alter (strictly speaking, lower) the onset of instability, (2) it may change the mode of oscillatory motion (e.g. from period-1 to period-2 motion), and (3) it may increase the amplitude of vibrations. Thus, studying the effects of time-dependent excitation on the dynamics and stability of lifting surfaces seems essential for their design. These studies are particularly crucial for the new generation of aircraft which are likely to be lighter, faster, more flexible, and more agile.

\section{REFERENCES}

[1] D. S. Woolston, H. L. Runyan, and R. E. Andrews, "An investigation ofeffects of certain types of structural nonlinearities on wing and controlsurface flutter," Journal of the Aeronautical Sciences, vol. 24 (1), 1957, pp.57-63.

[2] B. H. K. Lee, S. J. Price, and Y. S. Wong, "Nonlinear aeroelastic analysis ofairfoils: bifurcation and chaos," Progress in aerospace sciences, vol. 35 (3), 1999, pp. 205-334.

[3] E. H. Dowell and D. Tang, "Nonlinear aeroelasticity and unsteady aerodynamics,” AIAA journal, vol. 40 (9), 2002, pp. 1697-1707.

[4] D. C. Poirel and S. J. Price, "Post-instability behavior of a structurally nonlinear airfoil in longitudinal turbulence," Journal of Aircraft, vol. 34 (5), 1997, pp. 619-626.

[5] P. Marzocca, L. Librescu, and G. Chiocchia, "Aeroelastic response of 2-D lifting surfaces to gust and arbitrary explosive loading signatures," International Journal of Impact Engineering, vol. 25 (1), 2001, pp. 41-65.

[6] F. Haddadpour, S. Shams, and M. Kheiri, "Sharp edge gust effects on aeroelastic behavior of a flexible wing with high aspect ratio," in 43rd AIAA Aerospace Sciences Meeting and Exhibit, 2005, p. 838. 